\title{
Changes in Diffusion Metrics of the Red Nucleus in Chronic Stroke Patients With Severe Corticospinal Tract Injury: A Preliminary Study
}

\author{
Hanjun Kim, MD, Hoyoung Lee, MD, Kwang-Ik Jung, MD, PhD, \\ Suk Hoon Ohn, MD, PhD, Woo-Kyoung Yoo, MD, PhD
}

Department of Physical Medicine and Rehabilitation, Hallym University Sacred Heart Hospital, Anyang, Korea

\begin{abstract}
Objective To explore plastic changes in the red nucleus (RN) of stroke patients with severe corticospinal tract (CST) injury as a compensatory mechanism for recovery of hand function.

Methods The moderate group (MG) comprised 5 patients with synergistic hand grasp movement combined with limited extension, and the severe group (SG) included 5 patients with synergistic hand grasp movement alone. The control group (CG) included 5 healthy subjects. Motor assessment was measured by Motricity Index (MI). Diffusion tensor imaging was analyzed using fractional anisotropy (FA) and radial diffusivity (RD) in the individual regions of interest (ROIs) - bilateral internal capsule and anterior pons for CST injury and bilateral RN for rubrospinal tract (RST) injury.

Results The SG showed a significantly lower MI score than the MG mainly due to differences in hand subscores. Significantly reduced FA was observed in both MG and SG compared with CG, while SG showed increased MD and RD in the affected ROIs of CST, and increased FA on the unaffected side compared with CG. However, in the RN ROI, a significantly increased FA and decreased RD on the unaffected side similar to the affected side were found only in the SG. The relative index of FA was lower and RD in SG was higher than in CG in RST.

Conclusion The diffusion metrics of RST showed changes in patients with severe CST injury, suggesting that RST may play a role in the recovery of hand function in patients with severe CST injury.
\end{abstract}

Keywords Extrapyramidal tract, Neuronal plasticity, Recovery of function, Upper extremity, Paraplegia

\section{INTRODUCTION}

The corticospinal tract (CST) is a well-known major descending pathway connecting the brain and spinal cord in mammals. In primates, it is especially important for the control of hand movements, and considerable

Received June 19, 2017; Accepted September 11, 2017

Corresponding author: Woo-Kyoung Yoo

Department of Physical Medicine and Rehabilitation, Hallym University Sacred Heart Hospital, 22 Gwanpyeong-ro 170beon-gil, Dongan-gu, Anyang 14068, Korea. Tel: +82-31-380-3860, Fax: +82-31-380-3864, E-mail: mdwooky@gmail.com

ORCID: Hanjun Kim (http://orcid.org/0000-0001-8091-9110); Hoyoung Lee (http://orcid.org/0000-0001-7353-3113); Kwang-Ik Jung (http://orcid. org/0000-0003-1998-1921); Suk Hoon Ohn (http://orcid.org/0000-0002-1139-1946); Woo-Kyoung Yoo (http://orcid.org/0000-0002-1273-0647).

(c) This is an open-access article distributed under the terms of the Creative Commons Attribution Non-Commercial License (http://creativecommons.org/ licenses/by-nc/4.0) which permits unrestricted noncommercial use, distribution, and reproduction in any medium, provided the original work is properly cited. Copyright (c) 2018 by Korean Academy of Rehabilitation Medicine 
evidence points to its pivotal role in hand dexterity [1]. Damage to the CST is caused by various pathological conditions such as stroke and spinal cord injury. It often results in flaccid paralysis of the affected upper extremity. Hand is the most common and seriously affected area, as hand muscles are densely innervated by the CST for dexterous voluntary finger control [2-6]. In the few weeks following injury, many patients show partial recovery of function reflecting reorganization of the neural pathways [7]. Most previous studies focused on existing CST fibers for functional restoration of the hand [8]. However, studies related to the recovery of hand function involve mostly patients with mild-to-moderate weakness due to the methodological limitations in patients with severe paralysis [6,9-11]. Therefore, motor recovery in severe paralysis is a matter of debate that warrants investigation.

The rubrospinal tract (RST) is one of the descending extrapyramidal motor tracts closely related to limb movement in lower vertebrates $[12,13]$. RST facilitates muscle tone in the upper extremity while reaching and grasping with the activated hand flexor muscles in primates [14]. Red nucleus (RN) lesions interfere with several components of the reaching action, including aiming the limb using the proximal shoulder, elbow, and supination of the paw in animals [15-17]. A few animal [18-21] and human studies $[22,23]$ published recently have demonstrated plastic changes in the RST that compensate for CST injury. This functional redundancy between the RST and CST was documented decades ago in monkeys in the seminal series of studies by Lawrence and Kuyper [16,17], which reported improvements in reaching and grasp functions following complete bilateral pyramidotomy. These studies strongly suggest a potential compensatory role of RST in hand recovery of patients with severe CST injury, which has not been fully elucidated in humans.

Recent advances in diffusion tensor imaging (DTI) techniques have facilitated the investigation of connectivity and integrity of subcortical structures and fiber tracts based on water diffusion characteristics $[24,25]$. Recently, a method for measuring RST integrity in humans by studying healthy controls [26] and stroke patients [22,23] was published. Although, Jang and Kwon [23] described changes in RN connectivity in patients with relatively severe CST injury measured by CST tractography, their study included patients with moderate CST injury without a control group, which may be difficult to interpret as the influence of CST cannot be excluded.

We hypothesized that the degree of compensatory changes in the diffusion metrics of RST were greater in the presence of a higher degree of CST injury.

\section{MATERIALS AND METHODS}

\section{Subjects}

This study was conducted using a retrospective, controlled clinical trial design. Patients were recruited from inpatients in the rehabilitation department between January 2012 and July 2016 based on the following inclusion criteria: (1) history of chronic stroke, defined as stroke occurring for more than 6 months, (2) first-ever stroke, (3) middle cerebral artery (MCA) infarction or intracerebral hemorrhage (ICH) in the basal ganglia (BG), and (4) no demonstrable abnormal findings in the RST including RN on MRI. The exclusion criteria were: (1) operative management of stroke and (2) presence of other neurological diseases such as epilepsy or degenerative brain disease. A study was planned to compare three groups. Prior to a full-scale study, we conducted a preliminary study to elucidate the appropriate direction. A total of 15 participants were included and divided into three groups of 5 participants each. The moderate group (MG) comprised patients who showed synergistic hand grasp movement with partial extension of the affected hand, while the severe group (SG) comprised patients with partial synergistic hand grasp movement in the flexed-finger posture on the affected upper extremity. Five age-matched healthy subjects were enrolled as controls (CG).

\section{Resting motor threshold and motor evoked potential}

The stimulation set-up consisted of a Magnetic Stimulator STM 9000 (ATES MEDICA Device, Verona, Italy) for single pulse transcranial magnetic stimulation (TMS) intervention. A figure-8 coil was placed tangentially over the left primary motor cortex with the handle pointing at a $45^{\circ}$ angle posterolaterally. For the motor evoked potential (MEP) measurement, surface electromyography (EMG) was recorded using pre-gelled, disposable Ag/ $\mathrm{AgCl}$ electrodes with the active electrode in the contralateral first dorsal interosseous muscle, the reference electrode over the metacarpophalangeal joint, and the ground electrode over the wrist. The EMG signal was acquired at $3 \mathrm{kHz}$, filtered (10-500 Hz), amplified, and 
stored for offline analysis. The participants were seated in a comfortable chair with a headrest and their hands resting on their laps. The resting motor threshold, defined as the lowest stimulus intensity-evoked TMS-induced MEP of at least $50 \mathrm{mV}$ in 5 out of 10 consecutive trials in the target muscle $[27,28]$ was obtained over M1.

\section{DTI and lesion volume rendering}

DTI was acquired using a diffusion-weighted echoplanar imaging sequence $(\mathrm{TR}=5,000 \mathrm{~ms}$; $\mathrm{TE}=100 \mathrm{~ms}$; slice thickness=2.2 mm; no gap; in-plane resolution=2.4×2.4 $\mathrm{mm}, 45$ independent diffusion gradient directions using $\mathrm{b}=1,000 \mathrm{~s} / \mathrm{mm}^{2}$ ). DTI data were analyzed using FMRIB's Diffusion Toolbox from the FMRIB Software Library [13]. The region of interest (ROI) method was selected to measure tract integrity based on previous studies. Thus, RN is an appropriate part to draw ROI and the ROI method minimizes errors compared to the tract reconstruction method. We drew ROI on the posterior limb of internal capsule and the anterior pons on a color-coded map for CST and on the RN for RST individually. In each ROI, we extracted fractional anisotropy (FA), mean diffusivity (MD), axial diffusivity (AD), and radial diffusivity (RD). The ROI selection process is outlined in Fig. 1. A threedimensional reconstruction of the CST was performed for each patient in the MG and SG, and discontinuation of CST was found in all cases. To reduce motion artifacts of DTI, patients were instructed to maintain their head position during image acquisition. Furthermore, Eddy current correction was used to compensate for motion artifacts and distortion by the magnetic field.

Computer-assisted lesion volumes were acquired by MRIcro (http://people.cas.sc.edu/rorden/mricro/index. html).

\section{Clinical assessment}

The motor deficit was assessed using the Motricity Index (MI), an index ranging from 0 (hemiplegia) to 99 (no deficit). There are 33 points for hand pinch grip ( $0=$ no movement, 11 =beginning of prehension, 19=grips cube but unable to hold against gravity, 22=grips cube and holds against gravity but not against weak pull, 26=grips cube against pull but weaker than the other/normal side; 33=normal pinch grip). A score of 33 points each was assigned for elbow flexion and shoulder abduction ( $0=$ no movement, $9=$ palpable contraction in muscle but no movement, 14=movement seen but not full range/ not against gravity, $19=$ full range against gravity but not against resistance, $25=$ movement against resistance but weaker than the other side; 33=normal power) [29]. The O'Connor hand dexterity test, which measures smallpart manipulation ability, was also performed. This test requires the patient to place pins in a series of holes on a test board and the time for execution was measured [30], the Mini-Mental State Examination, which is a quick measure of cognitive function ranging from 0 (poor) to 30 (good), was performed to test cognition [31].

\section{Statistical analysis}

Statistical analysis was performed using SPSS version
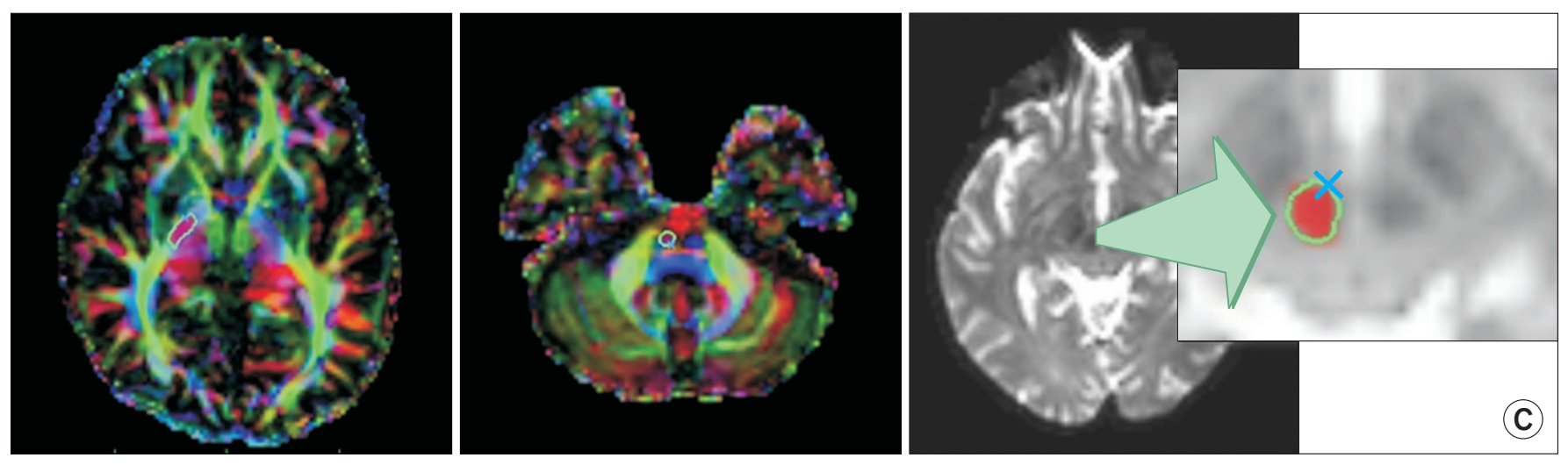

Fig. 1. Region of interest (ROI) selection. (A) Internal capsule ROI of the corticospinal tract. The posterior limb of the internal capsule within the projection fibers (dark and light blue color) was selected without including the retrosplenial fibers of internal capsule in the color-coded image. (B) Pons ROI of the corticospinal tract. The descending corticospinal tract at the pons level was drawn by selecting the anterior corticospinal tract (blue color) in the color-coded image. (C) The red nucleus. The dark area in the mid-brain was drawn on the T2 image. 
24.0 software (IBM, Armonk, NY, USA). The KruskalWallis test with Tukey's post hoc and Mann-Whitney U-test were used to evaluate the differences in clinical assessment and diffusion metrics of the three groups. We also calculated the relative index (RI) in the experimental (affected/unaffected side $\times 100$ ) and control (nondominant/dominant side $\times 100$ ) groups to evaluate the relative changes between the lesion and non-lesion sides $[32,33]$. To determine the MI, we not only obtained the total score of the affected upper extremity, but also the detailed subscores of shoulder abduction, elbow flexion, and hand pinch grasp to specifically evaluate the distal and proximal functions of the upper extremity. Statistical significance was defined as a $\mathrm{p}$-value of $<0.05$.

\section{RESULTS}

\section{Participant characteristics}

There were no statistically significant differences in the demographics of the three groups except for MI score. The MI score of SG was significantly lower than in MG. The MI subscores showed a statistically significant difference in hand pinch grasp but not in elbow flexion or shoulder abduction between the MG and SG. The O'Connor hand dexterity tests of the affected hand were not feasible in all patients due to poor hand function. Only a single patient in MG showed positive MEPs in the affected hemisphere, while no MEPs were evoked in other patients (Table 1).

\section{CST integrity measured by ROIs from internal capsule} and pons

In Kruskal-Wallis test for group comparison of CST diffusion metrics, the FA of both internal capsule ROI, the RD of the affected internal capsule ROI, and the FA of the affected pons ROI showed statistically significant differences. Post-hoc analysis revealed that in MG and SG, FAs of the affected internal capsule and pons ROIs were significantly lower than in CG $(\mathrm{p}<0.05)$. The FA of CST on the unaffected side was significantly higher than that of the CG alone in the internal capsule of patients in SG $(\mathrm{p}<0.05)$. Similarly, the MD and RD of CST on the affected side was significantly higher than in CG alone in the internal capsule of SG patients $(\mathrm{p}<0.05)$. Post-hoc analysis of the $\mathrm{AD}$ values of CST showed no statistically significant differences among the three groups. Kruskal-

Table 1. Demographic characteristics of control, moderate, and severe groups

\begin{tabular}{|c|c|c|c|c|}
\hline & $\begin{array}{c}\text { Control group } \\
(n=5)\end{array}$ & $\begin{array}{l}\text { Moderate group } \\
\quad(n=5)\end{array}$ & $\begin{array}{c}\text { Severe group } \\
(n=5)\end{array}$ & p-value \\
\hline Age (yr) & $48.9 \pm 8.5$ & $51.3 \pm 10.0$ & $50.8 \pm 1.9$ & 0.126 \\
\hline Gender & & & & 0.107 \\
\hline Men & 3 & 3 & 4 & \\
\hline Women & 2 & 2 & 1 & \\
\hline Lesion & & & & 0.094 \\
\hline Right & - & 1 & 2 & \\
\hline Left & - & 4 & 3 & \\
\hline MEP & & & & 0.092 \\
\hline Positive & - & 1 & 0 & \\
\hline Negative & - & 4 & 5 & \\
\hline Lesion volume $\left(\mathrm{cm}^{3}\right)$ & - & $7.97 \pm 1.04$ & $8.12 \pm 0.98$ & 0.118 \\
\hline MMSE & 30 & $28.4 \pm 1.41$ & $28.0 \pm 1.14$ & 0.604 \\
\hline \multicolumn{5}{|l|}{ Motricity Index } \\
\hline Total & 100 & $51.2 \pm 4.02 *$ & $32.8 \pm 4.91$ & 0.001 \\
\hline Shoulder+elbow & - & $31.0 \pm 2.73$ & $28.0 \pm 0.00$ & 0.070 \\
\hline Hand & - & $20.2 \pm 1.64^{*}$ & $4.8 \pm 4.91$ & 0.005 \\
\hline
\end{tabular}

Values are presented as mean \pm standard deviation or number.

MEP, motor evoked potential; MMSE, Mini-Mental State Examination.

${ }^{*} \mathrm{p}<0.05$. 
Table 2. Corticospinal tract diffusion metrics

\begin{tabular}{|c|c|c|c|c|c|c|c|c|}
\hline & \multirow[b]{2}{*}{$\begin{array}{l}\text { Control } \\
\text { group }\end{array}$} & \multicolumn{2}{|c|}{ Moderate group } & \multicolumn{2}{|c|}{ Severe group } & \multicolumn{3}{|c|}{ Relative index } \\
\hline & & Affected $^{\text {a) }}$ & Unaffected $^{\text {b) }}$ & Affected $^{\text {a) }}$ & Unaffected $^{\text {b) }}$ & $\begin{array}{l}\text { Control } \\
\text { group }\end{array}$ & $\begin{array}{c}\text { Moderate } \\
\text { group }\end{array}$ & $\begin{array}{l}\text { Severe } \\
\text { group }\end{array}$ \\
\hline \multicolumn{9}{|c|}{ Internal capsule } \\
\hline FA & $0.60 \pm 0.04$ & $0.19 \pm 0.05^{*}$ & $0.61 \pm 0.02$ & $0.15 \pm 0.08^{*}$ & $0.71 \pm 0.16^{*}$ & $0.98 \pm 0.07$ & $0.31 \pm 0.08^{*}$ & $0.23 \pm 0.16^{* * * *}$ \\
\hline MD & $0.70 \pm 0.02$ & $0.81 \pm 0.03$ & $0.72 \pm 0.03$ & $1.42 \pm 0.70^{*}$ & $0.59 \pm 0.14$ & $0.97 \pm 0.04$ & $1.12 \pm 0.29$ & $2.37 \pm 0.94^{*, * *}$ \\
\hline $\mathrm{AD}$ & $1.26 \pm 0.06$ & $0.96 \pm 0.26$ & $1.32 \pm 0.07$ & $1.61 \pm 0.74$ & $1.21 \pm 0.13$ & $0.96 \pm 0.05$ & $0.73 \pm 0.19 *$ & $1.29 \pm 0.49^{* * * *}$ \\
\hline $\mathrm{RD}$ & $0.41 \pm 0.03$ & $0.73 \pm 0.21$ & $0.41 \pm 0.02$ & $1.33 \pm 0.69 *$ & $0.43 \pm 0.15$ & $0.99 \pm 0.09$ & $1.73 \pm 0.44^{*}$ & $3.61 \pm 2.77^{*, * *}$ \\
\hline \multicolumn{9}{|l|}{ Pons } \\
\hline FA & $0.48 \pm 0.04$ & $0.32 \pm 0.10^{*}$ & $0.41 \pm 0.02$ & $0.33 \pm 0.08 *$ & $0.51 \pm 0.10$ & $0.96 \pm 0.04$ & $0.76 \pm 0.25^{*}$ & $0.66 \pm 0.17^{*}$ \\
\hline MD & $0.71 \pm 0.04$ & $0.75 \pm 0.08$ & $0.63 \pm 0.03$ & $0.77 \pm 019$ & $0.50 \pm 0.19$ & $1.06 \pm 0.04$ & $0.99 \pm 0.15$ & $1.13 \pm 0.06$ \\
\hline $\mathrm{AD}$ & $1.11 \pm 0.08$ & $0.98 \pm 0.07$ & $1.12 \pm 0.24$ & $1.03 \pm 0.06$ & $1.08 \pm 0.21$ & $1.04 \pm 0.04$ & $0.87 \pm 0.06$ & $0.95 \pm 0.07$ \\
\hline $\mathrm{RD}$ & $0.50 \pm 0.03$ & $0.63 \pm 0.11$ & $0.57 \pm 0.03$ & $0.64 \pm 0.17$ & $0.49 \pm 018$ & $1.08 \pm 0.06$ & $1.12 \pm 0.27$ & $1.38 \pm 0.27$ \\
\hline
\end{tabular}

Values are presented as mean \pm standard deviation.

FA, fractional anisotropy; $\mathrm{MD}$, mean diffusivity; $\mathrm{AD}$, axial diffusivity; RD, radial diffusivity.

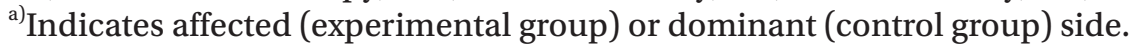

${ }^{b)}$ Indicates unaffected (experimental group) or non-dominant (control group) side.

${ }^{*} \mathrm{p}<0.05$ indicates a significant different from control group.

** $\mathrm{p}<0.05$ indicates a significant difference between moderate group and severe group.

(A)
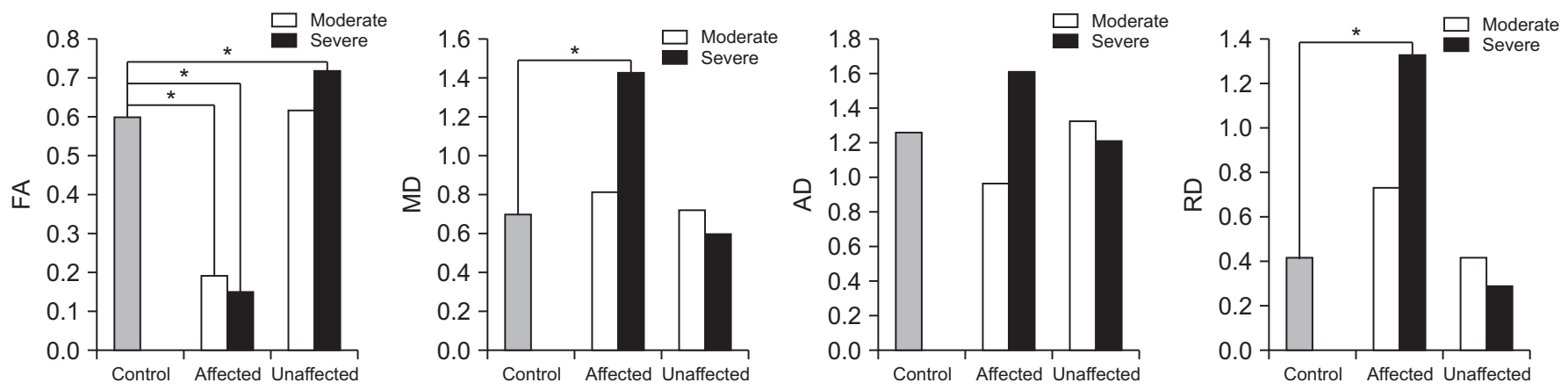

(B)
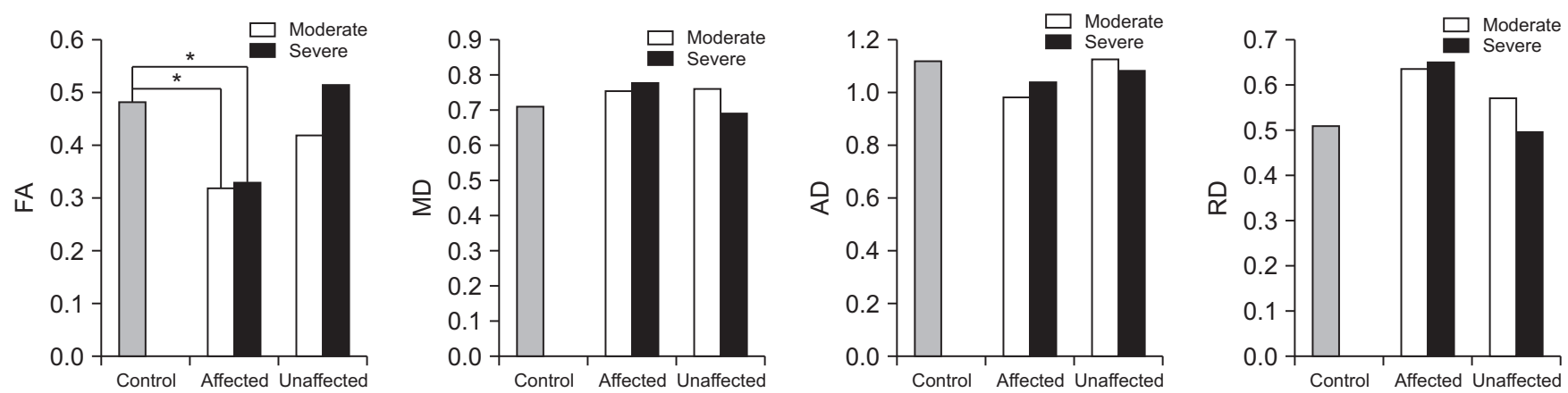

Fig. 2. Corticospinal metrics in the internal capsule (A) and pons (B) of the three groups. FA, fractional anisotropy; $\mathrm{MD}$, mean diffusivity; $\mathrm{AD}$, axial diffusivity; $\mathrm{RD}$, radial diffusivity. ${ }^{*} \mathrm{p}<0.05$. 
Wallis test for group comparisons of CST RI, RI of the FA, $\mathrm{MD}, \mathrm{AD}$, and $\mathrm{RD}$ in the internal capsule and pons ROI showed statistical significant differences. Post-hoc analysis revealed that RI of the FA in MG and SG were significantly lower than that of CG in the internal capsule and pons ROI, while the RI of MD, $\mathrm{AD}$, and RD in MG and SG were significantly higher than in CG specifically in the internal capsule ROI $(\mathrm{p}<0.05)$. Additionally, the RI of the $\mathrm{MD}, \mathrm{AD}$, and $\mathrm{RD}$ in SG were significantly higher and the RI of FA in SG was significantly lower than in MG, only in the internal capsule ROI ( $\mathrm{p}<0.05)$ (Table 2, Fig. 2).

\section{RST integrity measured by ROIs from RN}

In Kruskal-Wallis test for group comparison of RST diffusion metrics, the FA and RD of the unaffected side showed statistically significant differences. In post-hoc analysis, the FAs of RN on the unaffected side of RST in SG were significantly higher than those of CG. The RDs of RN on the unaffected side of RST in SG were significantly lower than in CG $(\mathrm{p}<0.05)$. The MD and AD values of RST showed no statistically significant differences among the three groups. In Kruskal-Wallis test for group comparisons of RI, the RI of FA and RD were significantly different. Post-hoc analysis revealed that RI of FA was lower and the RI of RD was higher in SG than in CG $(\mathrm{p}<0.05)$ (Table 3, Fig. 3).

\section{DISCUSSION}

In this study, we found significantly higher FA and lower RD in the unaffected RN ROI. A similar tendency in the affected RN ROI was observed in SG. Furthermore, the RI of RN showed statistically significant differences in FA and RD of SG, which indicates altered RN integrity following severe CST injury.

It is now widely accepted that the CST is important for voluntary control of hand and finger dexterity [1]. Therefore, lesions in CST interfere with the coordinated voluntary movement of the fingers resulting in functional impairment of the upper extremity. Several published

Table 3. Rubrospinal tract diffusion metrics

\begin{tabular}{|c|c|c|c|c|c|c|c|c|}
\hline & \multirow{2}{*}{$\begin{array}{l}\text { Control } \\
\text { group }\end{array}$} & \multicolumn{2}{|c|}{ Moderate group } & \multicolumn{2}{|c|}{ Severe group } & \multicolumn{3}{|c|}{ Relative index } \\
\hline & & Affected $^{\text {a) }}$ & Unaffected $^{\text {b) }}$ & Affec & Unaffected $^{\text {b) }}$ & $\begin{array}{c}\text { Control } \\
\text { group }\end{array}$ & $\begin{array}{l}\text { Moderate } \\
\text { group }\end{array}$ & $\begin{array}{l}\text { Severe } \\
\text { group }\end{array}$ \\
\hline FA & $0.41 \pm 0.09$ & $0.34 \pm 0.07$ & $0.46 \pm 0.17$ & $0.49 \pm 0.14$ & $0.64 \pm 0.16^{*}$ & $1.10 \pm 0.35$ & $0.80 \pm 0.24$ & $0.75 \pm 0.09 *$ \\
\hline MD & $0.60 \pm 0.03$ & $0.60 \pm 0.04$ & $0.63 \pm 0.05$ & $0.53 \pm 0.13$ & $0.50 \pm 0.13$ & $0.95 \pm 0.07$ & $0.95 \pm 0.05$ & $1.05 \pm 0.09$ \\
\hline $\mathrm{AD}$ & $0.88 \pm 0.10$ & $0.83 \pm 0.10$ & $0.98 \pm 0.11$ & $0.81 \pm 0.13$ & $0.92 \pm 0.12$ & $0.95 \pm 0.08$ & $0.85 \pm 0.09$ & $0.88 \pm 0.06$ \\
\hline $\mathrm{RD}$ & $0.40 \pm 0.05$ & $0.49 \pm 0.03$ & $0.46 \pm 0.12$ & $0.39 \pm 0.14$ & $0.29 \pm 0.15^{*}$ & $0.96 \pm 0.16$ & $1.14 \pm 0.33$ & $1.43 \pm 0.35^{*}$ \\
\hline
\end{tabular}

Values are presented as mean \pm standard deviation.

FA, fractional anisotropy; $\mathrm{MD}$, mean diffusivity; $\mathrm{AD}$, axial diffusivity; $\mathrm{RD}$, radial diffusivity.

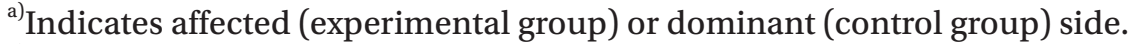

${ }^{b)}$ Indicates unaffected (experimental group) or non-dominant (control group) side.

${ }^{*} \mathrm{p}<0.05$ indicates a significant different from control group.
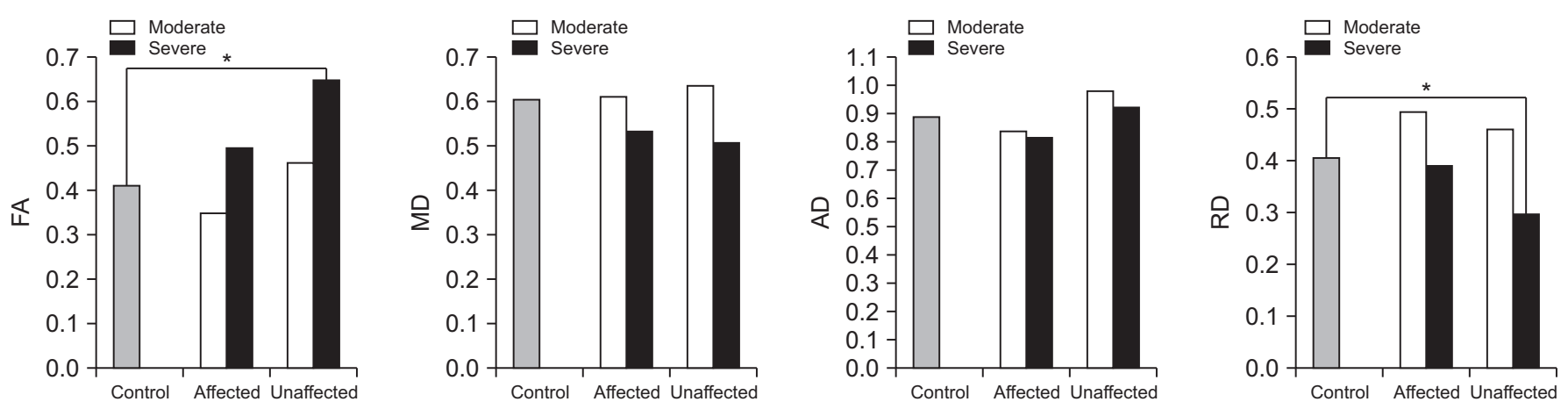

Fig. 3. Metrics of rubrospinal tract in the three groups. FA, fractional anisotropy; MD, mean diffusivity; AD, axial diffusivity; $\mathrm{RD}$, radial diffusivity. ${ }^{*} \mathrm{p}<0.05$. 
studies report the role of CST and motor recovery in patients with acute and subacute [7,8], chronic stroke [34] as well as in mild $[35,36]$ and severe motor impairments $[7,8]$. Despite the large amount of published evidence supporting the role of CST in hand function recovery, the relationship between hand function recovery and severe CST injury has rarely been studied [37]. The contralateral CST is recruited in severe injuries; however, the possibility of RST involvement in plasticity has yet to be extensively discussed due to the lack of an appropriate measurement tool or test in addition to the absence of an underlying conceptual hypothesis. Therefore, we studied only patients with severe chronic CST injury. All patients showed synergistic hand grasp movement, although partial extension was only observed in MG patients, indicating better CST integrity. Only a single patient showed MEPs in the affected hemisphere, indicating that patients in both SG and MG groups had severe damage involving the CST. The results of DTI for CST integrity supported this behavioral result. MD is the sum of $\mathrm{AD}$ and $\mathrm{RD}$. When the $\mathrm{AD}$ value is interpreted simply as axonal integrity and the $\mathrm{RD}$ value as myelin integrity, injury involving the MG may be interpreted as myelinopathy due to higher RD than $\mathrm{AD}$, while the injury in SG may be axonopathy as both the $\mathrm{AD}$ and $\mathrm{RD}$ values showed increasing tendency.

Lawrence and Kuypers $[16,17]$ documented the functional redundancy between the CST and RST by demonstrating relative preservation in reaching and hand grasp functions following bilateral pyramidotomy. Since then, a few animal [18-21] and human studies [22,23] reported compensatory changes in the RST following CST injury.

However, the changes in the microstructural integrity of RST in humans were only established by advances in DTI techniques in which various diffusion metrics were correlated with the function (FA value) of a specific tract and pathological status (MD, AD, RD) [38]. A few DTI studies observed changes in the RN following a stroke with CST injury in humans [22,23]. Takenobu et al. [22] investigated microstructural changes in the RST of patients with a mild subcortical stroke by conducting DTI three times in 2 weeks, and at 1 and 3 months. FA was higher in the affected RN than in the unaffected side only at 3 months based on tract-based spatial statistics [22]. However, the statistical power of this study was relatively low (uncorrected $\mathrm{p}<0.01$ ). In addition, it lacked clinical significance in patients with mild weakness, which is usually associ- ated with good recovery in CST plasticity. Another study by Jang et al [23] showed RN changes following striatocapsular hemorrhage, which was similar to our results. The study found increased FA and tract volume in the RN of the unaffected hemisphere compared with the affected hemisphere in patients with significant CST injury.

Despite the similarities in results, several differences need to be discussed. First, because we dealt with patients sustaining more severe CST injuries, we found significant changes in $\mathrm{RN}$ diffusion. Use of the entire tract volume rather than a single ROI should yield a larger value. Furthermore, we provided comparable normative data. Interestingly, although our patients were diagnosed with severe CST injury, the subscores of upper extremity MI indicate that MG and SG showed no significant differences in shoulder or elbow function, but only significantly reduced hand function in SG. This difference in hand subscores had the maximum effect on differences in the total upper extremity score. The CST is mainly distributed in the distal part of upper extremity, i.e., mostly the hand, which is specialized for selective control and dexterity, suggesting that the shoulder and elbow movement required for reaching is comparable in MG and SG. Higher levels of damage severity in CST integrity involving SG result in increased FA only in the RN to compensate for the reduced hand function.

Finally, the relationship between RST and CST from an evolutionary perspective offers interesting insights. Evidence derived from lower vertebrates suggests that the presence of RST is related to the presence of limbs [12,13]. The cellular composition of RN shows differences along the evolutionary hierarchy from quadrupedal lower vertebrates to bipedal primates such that the magnocellular portion of RN, which is a major component of RST in lower vertebrates, is comparatively small in primates [39]. Further, the presence of a transient well-developed magnocellular portion of $\mathrm{RN}$ involving the perinatal and developmental stages [40] suggests that RST plays an important role in the quadrupedal period. This portion of $\mathrm{RN}$ progressively changes as upper extremity function is increasingly adapted to the skilled movements controlled by CST and bipedalism. Following stroke-induced severe CST injury, triggering severe dysfunction of the hand, it is plausible that plasticity occurs in the functionally redundant areas of RST regulating the hand grasp function. Further studies involving the RST are warranted to dem- 
onstrate such plasticity.

Our study has a few limitations. First, because it is a preliminary study, it has a relatively small sample size without adequate statistical power. However, we recruited patients with severe CST injury and poor behavioral function, showing relatively large changes in RST and CST metrics compared with similar studies reported previously. Therefore, our study is considerably meaningful and is a stepping stone for larger studies in the future. Second, there was lesion heterogeneity. A few patients had BG ICH, while others were diagnosed with MCA infarction. However, because all patients sustained severe CST injuries based on both behavioral and objective measurements of the neural tract, we believe that the heterogeneity of lesions did not result in bias. Third, other factors such as initial severity of motor function and spasticity of limb that affect the outcomes were not investigated in this study. However, our study focused on chronic patients. Therefore, the authors believe that initial severity may not have a significant impact on the outcomes. Furthermore, this is a study investigating changes in RST integrity based on the degree of injury to CST, suggesting a low possibility that spasticity induced RST integrity. Nevertheless, these findings do not entirely exclude bias and further studies are needed to investigate such factors.

Despite the aforementioned limitations, this important study documents the changes in diffusion metrics associated with RN after severe CST injury. This study demonstrated that both the integrity of $\mathrm{RN}$ was increased in patients sustaining severe-to-complete CST injury. Therefore, further large-scale studies to address the current study limitations are warranted.

\section{CONFLICT OF INTEREST}

No potential conflict of interest relevant to this article was reported.

\section{REFERENCES}

1. Lemon RN. The G. L. Brown Prize Lecture: Cortical control of the primate hand. Exp Physiol 1993;78:263301.

2. Nakajima K, Maier MA, Kirkwood PA, Lemon RN. Striking differences in transmission of corticospinal excitation to upper limb motoneurons in two primate species. J Neurophysiol 2000;84:698-709.

3. Ehrsson HH, Fagergren A, Jonsson T, Westling G, Johansson RS, Forssberg H. Cortical activity in precision- versus power-grip tasks: an fMRI study. J Neurophysiol 2000;83:528-36.

4. Forssberg H. Neural control of human motor development. Curr Opin Neurobiol 1999;9:676-82.

5. Nowak DA, Grefkes C, Dafotakis M, Kust J, Karbe H, Fink GR. Dexterity is impaired at both hands following unilateral subcortical middle cerebral artery stroke. Eur J Neurosci 2007;25:3173-84.

6. Marshall RS, Perera GM, Lazar RM, Krakauer JW, Constantine RC, DeLaPaz RL. Evolution of cortical activation during recovery from corticospinal tract infarction. Stroke 2000;31:656-61.

7. Twitchell TE. The restoration of motor function following hemiplegia in man. Brain 1951;74:443-80.

8. Ward NS, Brown MM, Thompson AJ, Frackowiak RS. Neural correlates of motor recovery after stroke: a longitudinal fMRI study. Brain 2003;126(Pt 11):2476-96.

9. Ward NS, Newton JM, Swayne OB, Lee L, Thompson AJ, Greenwood RJ, et al. Motor system activation after subcortical stroke depends on corticospinal system integrity. Brain 2006;129(Pt 3):809-19.

10. Turton A, Wroe S, Trepte N, Fraser C, Lemon RN. Contralateral and ipsilateral EMG responses to transcranial magnetic stimulation during recovery of arm and hand function after stroke. Electroencephalogr Clin Neurophysiol 1996;101:316-28.

11. Boggio PS, Alonso-Alonso M, Mansur CG, Rigonatti SP, Schlaug G, Pascual-Leone A, et al. Hand function improvement with low-frequency repetitive transcranial magnetic stimulation of the unaffected hemisphere in a severe case of stroke. Am J Phys Med Rehabil 2006;85:927-30.

12. Kennedy PR. Corticospinal, rubrospinal and rubroolivary projections: a unifying hypothesis. Trends Neurosci 1990;13:474-9.

13. ten Donkelaar HJ. Evolution of the red nucleus and rubrospinal tract. Behav Brain Res 1988;28:9-20.

14.Zaaimi B, Edgley SA, Soteropoulos DS, Baker SN. Changes in descending motor pathway connectivity after corticospinal tract lesion in macaque monkey. Brain 2012;135(Pt 7):2277-89.

15. Whishaw IQ, Gorny B. Does the red nucleus pro- 
vide the tonic support against which fractionated movements occur? A study on forepaw movements used in skilled reaching by the rat. Behav Brain Res 1996;74:79-90.

16. Lawrence DG, Kuypers HG. The functional organization of the motor system in the monkey. I. The effects of bilateral pyramidal lesions. Brain 1968;91:1-14.

17. Lawrence DG, Kuypers HG. The functional organization of the motor system in the monkey. II. The effects of lesions of the descending brain-stem pathways. Brain 1968;91:15-36.

18. Alstermark B, Lundberg A, Pettersson LG, Tantisira B, Walkowska M. Motor recovery after serial spinal cord lesions of defined descending pathways in cats. Neurosci Res 1987;5:68-73.

19. Belhaj-Saif A, Cheney PD. Plasticity in the distribution of the red nucleus output to forearm muscles after unilateral lesions of the pyramidal tract. J Neurophysiol 2000;83:3147-53.

20. Perfiliev S, Pettersson LG, Lundberg A. Food-taking in the cat investigated with transection of the rubro- and corticospinal tracts. Neurosci Res 1998;32:181-4.

21. Pettersson LG, Blagovechtchenski E, Perfiliev S, Krasnochokova E, Lundberg A. Recovery of food-taking in cats after lesions of the corticospinal (complete) and rubrospinal (complete and incomplete) tracts. Neurosci Res 2000;38:109-12.

22. Takenobu Y, Hayashi T, Moriwaki H, Nagatsuka K, Naritomi H, Fukuyama H. Motor recovery and microstructural change in rubro-spinal tract in subcortical stroke. Neuroimage Clin 2013;4:201-8.

23. Jang SH, Kwon HG. Change of neural connectivity of the red nucleus in patients with striatocapsular hemorrhage: a diffusion tensor tractography study. Neural Plast 2015;2015:679815.

24. Assaf Y, Pasternak O. Diffusion tensor imaging (DTI)based white matter mapping in brain research: a review. J Mol Neurosci 2008;34:51-61.

25. Neil JJ. Diffusion imaging concepts for clinicians. J Magn Reson Imaging 2008;27:1-7.

26. Yang HS, Kwon HG, Hong JH, Hong CP, Jang SH. The rubrospinal tract in the human brain: diffusion tensor imaging study. Neurosci Lett 2011;504:45-8.

27. van Kuijk AA, Anker LC, Pasman JW, Hendriks JC, van Elswijk G, Geurts AC. Stimulus-response characteristics of motor evoked potentials and silent periods in proximal and distal upper-extremity muscles. J Electromyogr Kinesiol 2009;19:574-83.

28. Rossini PM, Barker AT, Berardelli A, Caramia MD, Caruso G, Cracco RQ, et al. Non-invasive electrical and magnetic stimulation of the brain, spinal cord and roots: basic principles and procedures for routine clinical application. Report of an IFCN Committee. Electroencephalogr Clin Neurophysiol 1994;91:79-92.

29. Demeurisse G, Demol O, Robaye E. Motor evaluation in vascular hemiplegia. Eur Neurol 1980;19:382-9.

30. Berger MA, Krul AJ, Daanen HA. Task specificity of finger dexterity tests. Appl Ergon 2009;40:145-7.

31. Dong Y, Sharma VK, Chan BP, Venketasubramanian N, Teoh HL, Seet RC, et al. The Montreal Cognitive Assessment (MoCA) is superior to the Mini-Mental State Examination (MMSE) for the detection of vascular cognitive impairment after acute stroke. J Neurol Sci 2010;299:15-8.

32. Basser PJ, Pierpaoli C. Microstructural and physiological features of tissues elucidated by quantitativediffusion-tensor MRI. J Magn Reson B 1996;111:20919.

33. Sun SW, Song SK, Hong CY, Chu WC, Chang C. Directional correlation characterization and classification of white matter tracts. Magn Reson Med 2003;49:2715.

34. Stinear CM, Barber PA, Smale PR, Coxon JP, Fleming MK, Byblow WD. Functional potential in chronic stroke patients depends on corticospinal tract integrity. Brain 2007;130(Pt 1):170-80.

35. Binkofski F, Seitz RJ, Arnold S, Classen J, Benecke R, Freund HJ. Thalamic metabolism and corticospinal tract integrity determine motor recovery in stroke. Ann Neurol 1996;39:460-70.

36. Carey LM, Abbott DF, Egan GF, Bernhardt J, Donnan GA. Motor impairment and recovery in the upper limb after stroke: behavioral and neuroanatomical correlates. Stroke 2005;36:625-9.

37. Butefisch CM, Kleiser R, Korber B, Muller K, Wittsack HJ, Homberg V, et al. Recruitment of contralesional motor cortex in stroke patients with recovery of hand function. Neurology 2005;64:1067-9.

38. Fillard P, Descoteaux M, Goh A, Gouttard S, Jeurissen B, Malcolm J, et al. Quantitative evaluation of 10 tractography algorithms on a realistic diffusion MR phantom. Neuroimage 2011;56:220-34. 
39. Hicks TP, Onodera S. The mammalian red nucleus and its role in motor systems, including the emergence of bipedalism and language. Prog Neurobiol 2012;96:165-75.
40. Yamaguchi K, Goto N. Development of the human magnocellular red nucleus: a morphological study. Brain Dev 2006;28:431-5. 